PROCEEDINGS OF THE

AMERICAN MATHEMATICAL SOCIETY

Volume 135, Number 10, October 2007, Pages 3295-3300

S 0002-9939(07)08861-2

Article electronically published on June 20, 2007

\title{
SMALE'S MEAN VALUE CONJECTURE AND THE COEFFICIENTS OF UNIVALENT FUNCTIONS
}

\author{
ANTHONY CONTE, EGE FUJIKAWA, AND NIKOLA LAKIC
}

(Communicated by Juha M. Heinonen)

\begin{abstract}
We study Smale's mean value conjecture and its connection with the second coefficients of univalent functions. We improve the bound on Smale's constant given by Beardon, Minda and Ng.
\end{abstract}

\section{INTRODUCTION AND RESULTS}

Let $P(z)$ be a complex polynomial of degree $d \geq 2$. Then $P(z)$ has $d-1$ critical points counting multiplicities. Denote the critical points by $z_{1}, z_{2}, \ldots, z_{d-1}$. If $z$ is not a critical point of $P$, then we have the following:

$$
\min _{j}\left|\frac{P(z)-P\left(z_{j}\right)}{z-z_{j}}\right| \leq 4\left|P^{\prime}(z)\right| .
$$

This inequality was first noted by Smale 8 . He also asked if the factor 4 in (1.1) can be replaced by $(d-1) / d$, which is the constant for the polynomial $P(z)=z^{d}+\lambda z$ $(\lambda \neq 0)$. He later repeated this problem in [7] and [9, and this is called Smale's mean value conjecture. The conjecture is true for $d=2,3,4$; see [11]. Furthermore, the case where $d=5$ was recently proved in 3 by using a method based on the results in 2]. The best known result for an arbitrary $d$ is found in [1, where the factor 4 in (1.1) is replaced by $4^{(d-2) /(d-1)}$. In this paper, we improve the estimate. Our main result is the following.

Theorem 1. Let $P$ be a polynomial of degree $d \geq 2$ and let $z_{1}, z_{2}, \ldots, z_{d-1}$ be the critical points of $P$. If $z$ is not a critical point of $P$, then

$$
\min _{j}\left|\frac{P(z)-P\left(z_{j}\right)}{z-z_{j}}\right| \leq 4 \frac{d-1}{d+1}\left|P^{\prime}(z)\right| .
$$

Let

$$
S(P, z)=\min _{j}\left|\frac{P(z)-P\left(z_{j}\right)}{\left(z-z_{j}\right) P^{\prime}(z)}\right|
$$

Received by the editors June 2, 2005 and, in revised form, July 18, 2006.

2000 Mathematics Subject Classification. Primary 30C10; Secondary 30C50.

Key words and phrases. Polynomial, critical point, univalent function.

The third author was partially supported by the grant DMS 0200733 from the National Science Foundation.

(C)2007 American Mathematical Society Reverts to public domain 28 years from publication 
The quotient is invariant under pre- and post- compositions of affine maps; specifically if

$$
Q(w)=\frac{P(z+a w)-P(z)}{a P^{\prime}(z)},
$$

then $Q(0)=0, Q^{\prime}(0)=1$ and $w_{j}=\left(z_{j}-z\right) / a$ are the critical points of $Q(w)$. Therefore,

$$
\left|\frac{Q\left(w_{j}\right)}{w_{j}}\right|=\left|\frac{P(z)-P\left(z_{j}\right)}{\left(z-z_{j}\right) P^{\prime}(z)}\right| .
$$

Thus we may assume that $P(z)$ in (1.1) and (1.2) fixes $z=0$ and has derivative 1 at 0 . Furthermore, we may assume that $\min _{j}\left|w_{j}\right|=\min _{j}\left|\left(z_{j}-z\right) / a\right|$ is obtained when $j=1$, and taking

$$
a=e^{i \theta} \frac{\min _{j}\left|P(z)-P\left(z_{j}\right)\right|}{\left|P^{\prime}(z)\right|} \quad \text { with } \quad \theta=-\arg \left(z-z_{1}\right)
$$

makes $w_{1}$ positive and $\min _{j}\left|Q\left(w_{j}\right)\right|=\min _{j}\left|P(z)-P\left(z_{j}\right)\right|=1$. All this implies that

$$
S(P, z)=S(Q, 0) \leq \frac{1}{w_{1}} .
$$

We summarize as the following definition.

Definition 1. A polynomial $P(z)$ of degree $d \geq 2$ with critical points $z_{1}, z_{2}, \ldots$, $z_{d-1}$ is said to be normalized if the following conditions are satisfied: $P(0)=0$, $P^{\prime}(0)=1, \min _{j}\left|P\left(z_{j}\right)\right|=1$ and $\min _{j}\left|z_{j}\right|=z_{1}>0$.

Let $f$ be the inverse branch of a normalized polynomial $P$ satisfying $f(0)=0$. Then $f$ is in the class $S$, that is, the set of all normalized univalent functions on the open unit disc $\Delta$. By applying the Koebe covering theorem to $f$, we see that every critical point $z_{j}$ of $P$ satisfies $\left|z_{j}\right| \geq 1 / 4$. Thus

$$
\min _{j}\left|\frac{P\left(z_{j}\right)}{z_{j}}\right| \leq \max _{j} \frac{1}{\left|z_{j}\right|}=\frac{1}{z_{1}} \leq 4,
$$

which proves (1.1).

Let

$$
C(d):=\sup _{P} \frac{1}{z_{1}},
$$

where the supremum is taken over all normalized polynomials $P$ of degree $d$. Then

$$
S(P, z) \leq C(d) .
$$

Thus any upper bound on $C(d)$ less than 4 is obviously an improvement on the constant 4 in (1.1).

By using estimates for the coefficients of univalent functions, we have the following theorem.

Theorem 2. Let $d \geq 2$. Then

$$
C(d) \leq 4 \frac{d-1}{d+1} .
$$

By the above consideration, Theorem 2 implies Theorem 1.

Our method for the proof of Theorem 2 will be applied to some special cases. The first one is an extension of the theorem of $\mathrm{Ng}[6]$ : 
Corollary 1. If a normalized polynomial $P$ satisfies $P^{\prime \prime}(0)=0$, then

$$
S(P, 0) \leq 2 .
$$

Corollary 2. If all the critical points of a normalized polynomial $P$ lie in the right half-plane, then

$$
S(P, 0) \leq \frac{4}{3}
$$

In Section 2, we prove Theorem 2 and the corollaries. In Section 3, we summarize a relationship between the various constants associated with Smale's mean value conjecture.

The authors would like to thank the referee for many important suggestions. We would also like to thank Edward Crane for his helpful comments.

\section{UNIVALENT FUNCTIONS AND THE CRITICAL POINTS}

Let $P(z)$ be a normalized polynomial of degree $d \geq 2$ and let $z_{1}, z_{2}, \ldots, z_{d-1}$ be the critical points of $P(z)$. Then the derivative of $P(z)$ is

$$
P^{\prime}(z)=\left(1-\frac{z}{z_{1}}\right)\left(1-\frac{z}{z_{2}}\right) \cdots\left(1-\frac{z}{z_{d-1}}\right)
$$

and by a simple integration, we have

$$
P(z)=z-\left(\frac{1}{2} \sum_{i=1}^{d-1} \frac{1}{z_{i}}\right) z^{2}+\left(\frac{1}{3} \sum_{i \neq j}^{d-1} \frac{1}{z_{i} z_{j}}\right) z^{3}-\cdots+\frac{(-1)^{d-1}}{d \cdot z_{1} z_{2} \cdots z_{d-1}} z^{d}
$$

Let $f(w)=w+a_{2} w^{2}+\cdots$ be the inverse branch of $P$ with $f(0)=0$. Since $f$ is in the class $S$, we have $\left|a_{2}\right| \leq 2$ by the area theorem (see [4] and [5]). Observe that none of the $z_{i}$ are in the image $f(\Delta)$. Then the function

$$
f_{i}(w)=\frac{f(w)}{1-f(w) / z_{i}}
$$

is also in the class $S$, and the coefficient of $w^{2}$ in the expansion of $f_{i}$ is $a_{2}+1 / z_{i}$. Therefore

$$
\left|a_{2}+\frac{1}{z_{i}}\right| \leq 2 \quad(i=1, \ldots, d-1)
$$

by the area theorem again. In particular,

$$
\left|a_{2}+\frac{1}{z_{1}}\right| \leq 2
$$

and by the triangle inequality,

$$
\left|\frac{1}{z_{i}}-\frac{1}{z_{1}}\right| \leq 4 .
$$

Our estimate can now be derived as follows.

Proof of Theorem 2. Since $f(P(z))=z$, it follows that $-P^{\prime \prime}(0)=f^{\prime \prime}(0)=2 a_{2}$. Then by (2.1),

$$
a_{2}=\frac{1}{2} \sum_{i=1}^{d-1} \frac{1}{z_{i}}
$$


which we substitute into (2.3) to get

$$
\left|\frac{1}{2} \sum_{i=1}^{d-1} \frac{1}{z_{i}}+\frac{1}{z_{1}}\right| \leq 2 .
$$

We take the real part of the above inequality to find

$$
\frac{3}{z_{1}}+\sum_{i=2}^{d-1} \operatorname{Re} \frac{1}{z_{i}} \leq 4
$$

Taking the real part of (2.4) gives

$$
\frac{1}{z_{1}}-4 \leq \operatorname{Re} \frac{1}{z_{i}}
$$

Substituting this inequality for the remaining terms in (2.6) yields

$$
\frac{3}{z_{1}}+(d-2)\left(\frac{1}{z_{1}}-4\right) \leq 4
$$

which is easily solved for $1 / z_{1}$. Therefore

$$
C(d)=\sup _{P} \frac{1}{z_{1}} \leq 4 \frac{d-1}{d+1}
$$

which completes a proof.

Certain restrictions on polynomials permit modifications of the proof of Theorem 2. If $P^{\prime \prime}(0)=0$, then $a_{2}=0$. Applying this to (2.3), we have

$$
S(P, 0) \leq \frac{1}{z_{1}}=\left|a_{2}+\frac{1}{z_{1}}\right| \leq 2,
$$

and Corollary 1 is proved.

Next we prove Corollary 2. Suppose that all the critical points lie in the right half-plane. Then all the quantities in the sum in (2.6) are positive, and we have

$$
\frac{3}{z_{1}} \leq 4
$$

which yields the assertion.

\section{VARIOUS CONSTANTS}

Consider a normalized polynomial $P(z)$ with critical points $z_{1}, z_{2}, \ldots, z_{d-1}$. Then $\min _{j}\left|P\left(z_{j}\right)\right|=1$ and $\min _{j}\left|z_{j}\right|=z_{1}>0$. Let $i:=i(P)$ be an index satisfying $\left|P\left(z_{i(P)}\right)\right|=1$. Then

$$
\min _{j}\left|\frac{P\left(z_{j}\right)}{z_{j}}\right| \leq\left|\frac{P\left(z_{i(P)}\right)}{z_{i(P)}}\right|=\frac{1}{\left|z_{i(P)}\right|} .
$$

We choose $i(P)$ so that $\left|z_{i(P)}\right|$ is as large as possible and we define

$$
N(d)=\sup _{P} \frac{1}{\left|z_{i(P)}\right|},
$$

where the supremum is taken over all normalized polynomials $P$ of degree $d$. Since $\left|z_{1}\right| \leq\left|z_{i(P)}\right|$, we have

$$
N(d) \leq C(d) .
$$


We conjecture that $C(d) \leq 1$. The factor 4 in (1.1) is the inverse of the Koebe constant 1/4. Tishler asked in [10] if the covering constant for the class of normalized inverse branches is larger than $1 / 4$. Let $\kappa$ be this constant. Then $\kappa=\inf _{d} \kappa(d)$, where $\kappa(d)$ is the covering constant for the class of normalized inverse branches of polynomials of degree $d$. Since $\left|z_{j}\right| \geq \kappa(d)$ for any critical point $z_{j}$, the following sequence of inequalities holds:

$$
S(P, z) \leq N(d) \leq C(d) \leq \frac{1}{\kappa(d)} .
$$

Note that each constant represents different problems. The Koebe covering theorem implies that

$$
S(P, z) \leq N(d) \leq C(d) \leq \frac{1}{\kappa} \leq 4
$$

The referee of this paper points out that our approach may be used to show that

$$
\kappa(d) \geq \frac{d+1}{4 d} .
$$

To see this, observe that, for $f \in S$ and for $w \notin f(\Delta)$, the proof of inequalities (2.2) together with the triangle inequality implies that

$$
|w| \geq \frac{1}{2+\left|a_{2}\right|} .
$$

Furthermore, by inequalities (2.2), we have

$$
\left|\sum_{i=1}^{d-1}\left(a_{2}+\frac{1}{z_{i}}\right)\right| \leq \sum_{i=1}^{d-1}\left|a_{2}+\frac{1}{z_{i}}\right| \leq 2(d-1) .
$$

On the other hand, (2.5) implies that

$$
\left|\sum_{i=1}^{d-1}\left(a_{2}+\frac{1}{z_{i}}\right)\right|=\left|(d-1) a_{2}+2 a_{2}\right| .
$$

Thus we obtain

$$
\left|a_{2}\right| \leq 2 \frac{d-1}{d+1}
$$

Therefore,

$$
|w| \geq \frac{1}{2+\left|a_{2}\right|} \geq \frac{d+1}{4 d} .
$$

This yields that

$$
S(P, z) \leq N(d) \leq C(d) \leq \frac{1}{\kappa(d)} \leq \frac{4 d}{d+1},
$$

while our main result of this paper can be stated as

$$
S(P, z) \leq N(d) \leq C(d) \leq 4 \frac{d-1}{d+1} .
$$




\section{REFERENCES}

[1] Beardon, A. F., Minda, D. and Ng, T. W., Smale's mean value conjecture and the hyperbolic metric, Math. Ann. 322 (2002), 623-632. MR1905109 (2003d:30005)

[2] Crane, E. T., Extremal polynomials in Smale's mean value conjecture, Comput. Methods Funct. Theory. 6 (2006), 145-163. MR2241037

[3] Crane, E. T., A computational proof of the degree 5 case of Smale's mean value conjecture, preprint.

[4] Duren, P., Univalent functions, Springer-Verlag, 1983. MR708494 (85j:30034)

[5] Henrici, P. and Kenan, W. R., Applied and Computational Complex Analysis, Vol. 3. Wiley, 1994. MR $822470(87 \mathrm{~h}: 30002)$

[6] Ng, T. W., Smale's mean value conjecture for odd polynomials, J. Aust. Math. Soc. 75 (2003), 409-411. MR2015325 (2004j:30013)

[7] Shub, M. and Smale, S., Computational complexity: On the geometry of polynomials and the theory of cost. II, SIAM J. Comput. 15 (1986), 145-161. MR822199 (87m:68044)

[8] Smale, S., The fundamental theorem of algebra and complexity theory, Bull. Amer. Math. Soc. (N. S.) 4 (1981), 1-36. MR.590817 (83i:65044)

[9] Smale, S., Mathematical Problems for the Next Century, Mathematics: frontiers and perspectives, eds. Arnold, V., Atiyah, M., Lax, P. and Mazur, B., Amer. Math. Soc., 2000. MR1754783 (2001i:00003)

[10] Tischler, D., Perturbations of critical fixed points of analytic maps, Complex analytic methods in dynamical systems (Rio de Janeiro, 1992) Astérisque 222 (1994), 407-422. MR1285398 (95k:30003)

[11] Sheil-Small, T., Complex polynomials, Cambridge Studies in Advanced Mathematics 75, Cambridge, 2002. MR1962935 (2004b:30001)

Department of Mathematics, Graduate Center, CUny, New York, New York 10016 E-mail address: ajconte520@hotmail.com

Department of Mathematical and Computing Sciences, Tokyo Institute of Technology, Oh-OKayama, Meguro-Ku, TOKYo, 152-8552 Japan

Current address: Department of Mathematics, Sophia University, 7-1 Kioi-cho, Chiyoda-ku, Tokyo, 102-8554 Japan

E-mail address: fujikawa@m.sophia.ac.jp

Department of Mathematics and Computer Science, Herbert H. Lehman College, CUNY, BRonx, New York 10468

E-mail address: nikola.lakic@lehman.cuny.edu 